\title{
Simultaneous optical pulse compression and wing reduction
}

\author{
N. J. Halas ${ }^{a)}$ and D. Grischkowsky \\ IBM Watson Research Center, Yorktown Heights, New York 10598
}

(Received 21 November 1985; accepted for publication 3 February 1986)

\begin{abstract}
We report the compression of picosecond optical pulses with a simultaneous reduction of the pulse wings by using a combination of both the self-phase modulation and nonlinear birefringence effects in a modified optical-fiber pulse compressor.
\end{abstract}

Optical pulse compression via the optical-fiber pulse compressor ${ }^{1}$ has become an important experimental technique to achieve shorter pulses for many different applications and at a variety of wavelengths. The operation of the pulse compressor is based on the self-phase modulation effect which increases the bandwidth of the input pulse and which together with the group velocity dispersion of the fiber produces the frequency sweep (chirp) that makes compression with the subsequent dispersive delay line possible. A recent example was that by using two-stage optical pulse compression together with amplification, the 5 ps pulses from a synchronously pumped, mode-locked dye laser were compressed to only $16 \mathrm{fs} .^{2}$ Other examples include the compression of the $33 \mathrm{ps}, 0.53 \mu \mathrm{m}$ second harmonic pulses (generated by a mode-locked YAG laser) to $0.4 \mathrm{ps}^{3}{ }^{3}$ and the compression of the $80 \mathrm{ps}, 1.06 \mu \mathrm{m}$ fundamental output pulses to $1.8 \mathrm{ps}^{4}$ and then to $1.3 \mathrm{ps}^{5}{ }^{5}$ In addition, the shortest optical pulses have for some time been produced by compressing the pulses from the colliding pulse mode-locked ring dye laser. ${ }^{6-9}$

However, for the above examples of pulse compression, the compressed pulses had relatively broad low power wings, which can be detrimental to energy integrating measurements. One method of eliminating these wings is based on the nonlinear birefringence effect in single-mode optical fibers. ${ }^{10-13}$ This effect causes a different amount of intensitydependent phase to be acquired by the field components along the two axes of the optical fiber. The consequent phase difference $\phi(t)$ between the components causes a change in the state of the output polarization. For sufficient intensity, the output polarization becomes strongly intensity dependent. Therefore, if a polarizer at the output end of the fiber, in conjunction with a Soleil-Babinet compensator, is set to block the low intensity light it will operate to transmit the peak of the pulse and attenuate the pulse wings. For this simplest mode of operation the output pulse shape is proportional to the cube of the input pulse shape. This application was first discussed by Stolen et al., ${ }^{10}$ and was demonstrated for picosecond pulses in the visible by Nikolaus et al. ${ }^{11}$ and with soliton propagation in the infrared by Mollenauer et al. ${ }^{12}$ Unfortunately, for subpicosecond pulses, with reasonable phase differences $\phi(t)$, the accompanying self-phase modulation becomes so strong that the pulses are severely broadened by group-velocity dispersion, and this approach can no longer be used. Another method of wing clipping recently introduced by Heritage et al. ${ }^{5}$ involves spectral windowing of a pulse in the optical-fiber pulse compressor. This

s' Bryn Mawr College, Bryn Mawr, Pennsylvania. method eliminates the spectral components at the edges of the spectral envelope that do not compress well in the subsequent dispersive delay line.

In this letter we report the usage of both self-phase modulation and nonlinear birefringence to achieve optical pulse compression together with optical pulse reshaping on the subpicosecond time scale in a modified optical-fiber pulse compressor. We have compressed the 6 ps pulses from a mode-locked dye laser to $0.38 \mathrm{ps}$; simultaneously, we have severely clipped the wings of this pulse so that it compares favorably with a (sech) ${ }^{2}$ pulse shape.

We will now describe our experimental method and results in detail. The experimental setup is as shown in Fig. 1. The commercial laser source was a synchronously pumped, mode-locked tunable dye laser, ${ }^{1}$ whose output at $5900 \AA$ consisted of 6 ps FWHM pulses, cavity dumped at a repetition rate of $4 \mathrm{MHz}$. A variable disk attenuator was placed permanently in the beam to attenuate the incident light yet not interfere with the coupling efficiency into the optical fiber. The input beam was then transmitted through a crystal polarizer to ensure a high degree of linear polarization. A polarization rotator (half-wave plate) was used to vary the polarization angle of the beam incident on the optical fiber. The light was then coupled with $40 \%$ efficiency into five meters of ITT- 1601 nonpolarization-preserving single-mode optical fiber, by means of a Newport fiber coupler ${ }^{14}$ and a $10 \times$ microscope objective lens. The fiber had a core diameter of $4 \mu \mathrm{m}$; the nonlinear index $n_{2}$ and the group velocity dispersion (GVD) were that of fused quartz. ${ }^{1.15}$ The fiber was loosely wound on a $20-\mathrm{cm}$-diam spool. The beat length of the mounted fiber was of the order of its $5 \mathrm{~m}$ length, and this value was relatively insensitive to temperature changes. However, if the fiber was moved, the linear birefringence and the axes of the fiber changed, ${ }^{16}$ but on a daily basis this experimental setup for the fiber was quite stable. At the fiber output, the beam, recollimated by another $10 \times$ objective lens, was passed through a Soleil-Babinet compensator to cancel any acquired phase shift in the fiber due to its small linear birefringence, and then passed through the output polarizer, which blocked the low intensity light. Another polarization rotator optimized the diffraction efficiency for the dispersive delay line, consisting of a $1800 \mathrm{line} / \mathrm{mm}$ holographic grating, and a right angle prism. The optimum separation between the grating and prism was $13 \mathrm{~cm}$. The autocorrelation traces were obtained by noncollinear generation of second harmonic light in a $200-\mu$ m-thick KDP crystal; the time delay was scanned by a computer-driven stepping motor synchronized with a multichannel analyzer used in the signal averaging mode. Following a procedure similar to 


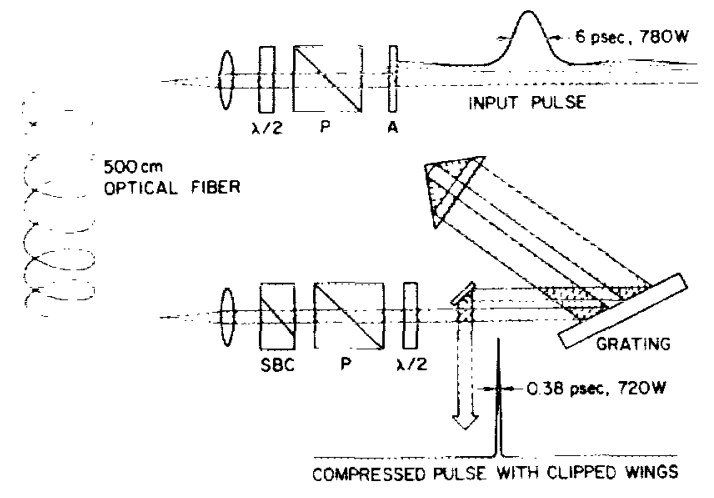

FIG. 1. Schematic diagram of experiment. $A$ indicates the variable attenuator; $P$ indicates a crystal polarizer; $\lambda / 2$ indicates a half-wave plate; SBC indicates the Soleil-Babinet compensator.

that described in Ref. 2, electronic pulse selection was employed to minimize the effect of laser pulse fluctuations.

Figure 2 is a plot of the average output power transmitted through the crossed output polarizer as a function of the average input power. The characteristic curve is nearly cubic over more than an order of magnitude. For low levels of input light the slope is less than cubic, and at the lowest levels the slope becomes linear. This is due to a slight linear depolarization of the light upon propagation through the fiber, which at very low power levels is responsible for the transmitted power. For this case, the measured polarization ratio for the output light from the optical fiber was consistently less than 1/1000. The cubic region of the curve (and below) corresponds to a maximum induced phase difference $\phi_{m}<\pi$ at the peak of the pulse. Above this cubic regime (for higher input powers than shown in Fig. 2) the curve again acquires a subcubic slope. In this region $\phi_{m}>\pi$, and the curve saturates because the maximum transmission no longer occurs at the peak of the pulse. In this region of operation we have observed a three-peaked pulse autocorrelation corresponding to a dip in the center of the transmitted pulse.

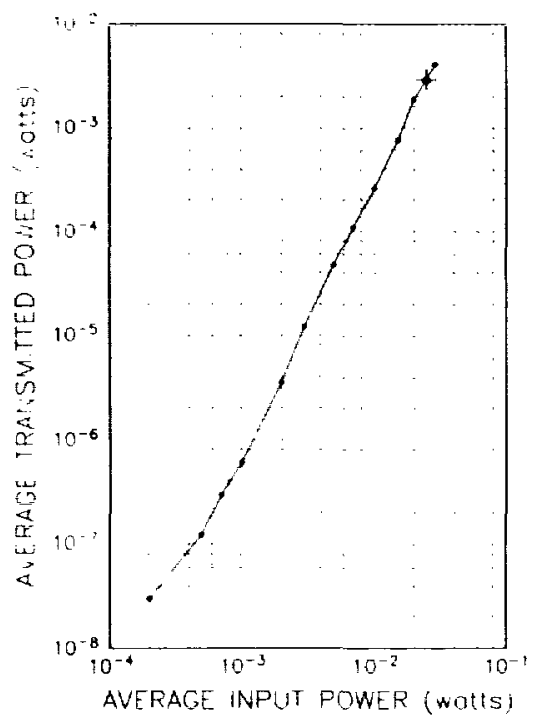

FIG. 2. Measured characteristic curve
The strength of the nonlinear birefringence depends on the angle of the input polarization with respect to the effective fiber axes. ${ }^{16}$ In the cubic regime of the characteristic curve we have measured a factor of three change in the output power as this angle was varied. The maximum and minimum values occurred with the polarization along the effective axes. These results disagree with the theories of Refs. 10 and 13. Both theories predict no effect when the polarization is aligned along an axis of the fiber. Reference 10 neglects linear birefringence, the effect of which was later analyzed in Ref. 13. Both theories neglect circular birefringence (optical activity) which is induced by twists in the fiber. ${ }^{17}$ The inclusion of circular birefringence weakens the strong angular dependence of the nonlinear birefringence as predicted above. However, the self-phase modulation effect does not show any significant angular dependence.

In order to optimize the system for simultaneous pulse compression and wing clipping the following procedure is recommended. The length of the fiber is determined in the ordinary way for pulse compression as described in Refs. 1 and 18 , although for this application we recommend nonpolarization preserving fiber. ${ }^{19}$ To properly optimize the rotation, characteristic curves such as Fig. 2 should be taken as a function of angle with respect to the fiber axes. One then adjusts the strength of the nonlinear birefringence to obtain a polarization change of $90^{\circ}\left(\phi_{m}=\pi\right)$ at the peak of the pulse for the maximum possible input power. This procedure gives the best throughput with the maximum bandwidth. For the results shown in Figs. 2 and 3, the input polarization was aligned along the axis for which the nonlinear birefringence was a minimum. An added advantage was that this situation eliminated the need for the Soleil-Babinet compensator.
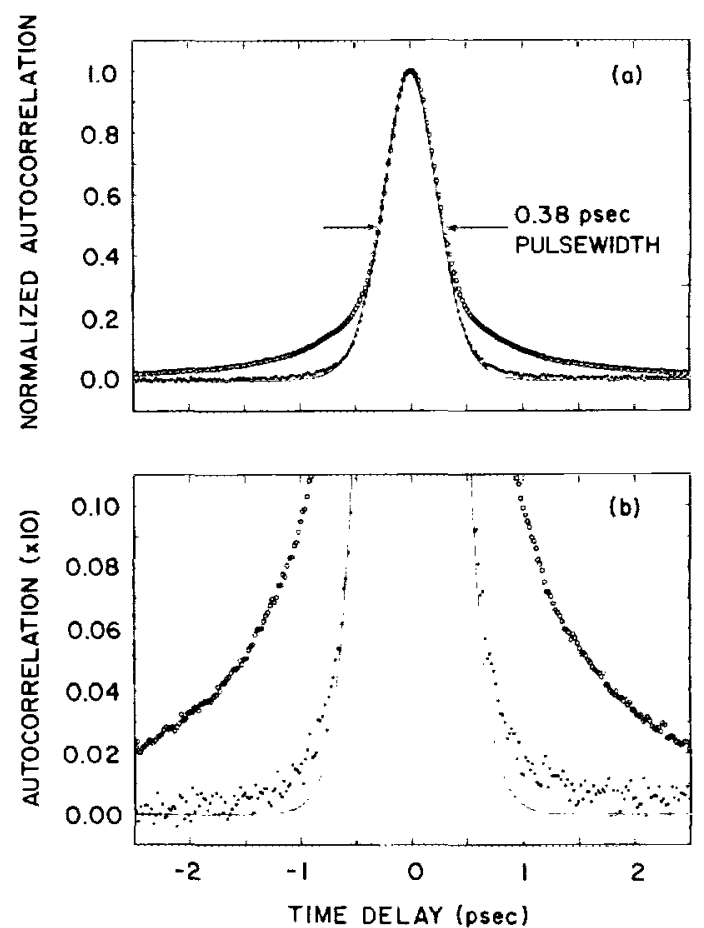

FIG. 3. (a). Autocorrelation measurements. Compressed pulse obtained using this technique (points); standard compressed pulse (open circles); calculated autocorrelation of $(\operatorname{sech})^{2}$ (solid line). (b) $10 \times$ magnified view of (a). 
The cross on the characteristic curve marks the conditions for the experimental results shown in Fig. 3. The indicated $25 \mathrm{~mW}$ average input power yielded an average output power from the polarizer of $2.8 \mathrm{~mW}$, for a final average output power from the compressor of $1.1 \mathrm{~mW}$. One can understand this $2.8 \mathrm{~mW}$ output power in terms of the following considerations. The coupling efficiency of $40 \%$ gave $10 \mathrm{~mW}$ of power coupled into the fiber. Since we have separately measured our laser pulses by cross correlation with compressed pulses, we know that approximately $1 / 4$ of the energy of the laser output pulses resided in satellite pulses and low level wings that surrounded the main pulse. Consequently, only $7.5 \mathrm{~mW}$ of the coupled power was in the pulses that drove the nonlinear effects of self-phase modulation and nonlinear birefringence. Knowing the $4 \mathrm{MHz}$ repetition rate and the input pulse width of $6 \mathrm{ps}$, we calculate that the driving pulses in the fiber had peak powers of $310 \mathrm{~W}$. If we assume that the switching efficiency was $100 \%$ at the peak of these pulses $\left(\phi_{m}=\pi\right)$, and because the transmitted pulse shape is proportional to $\sin ^{2}(\phi / 2),{ }^{10,11}$ the transmitted energy would be $60 \%$ for our typical laser pulse shapes. Thus, for $\phi_{m}=\pi$, the transmission of the $7.5 \mathrm{~mW}$ average power of the $310 \mathrm{~W}$ driving pulses would be $60 \%$ giving the predicted average output power of $4.5 \mathrm{~mW}$ compared to the measured value of $2.8 \mathrm{~mW}$. This difference indicates that $\phi_{m}$ was approximately $0.7 \pi$ for our measurement. The loss in the dispersive delay line was mainly due to the $65 \%$ diffraction efficiency of the grating. The $1.1 \mathrm{~mW}$ average compressed power corresponds to a peak power of $720 \mathrm{~W}$ for the $380 \mathrm{fs}$ compressed pulses.

The intensity autocorrelation measurement of the compressed and reshaped optical pulses is shown in Fig. 3. For this result the separation between the grating and prism was optimized for the shortest pulses with the cleanest pulse shapes. The measured bandwidth for these compressed pulses was $35 \mathrm{~cm}^{-1}$, to be compared to $29 \mathrm{~cm}^{-1}$ required for a transform limited (sech) ${ }^{2}$ pulse shape with the same pulse width. For comparison a standard compressed pulse shape (normalized to the same pulse width) is also shown. One sees a marked reduction in the wings of the optical pulses obtained via this new technique. Also, one sees that with this method of pulse compression/reshaping the resulting pulses compared favorably to the calculated (sech) ${ }^{2}$ autocorrelation shown as the solid line.

In conclusion, we have demonstrated an optical pulse compression and reshaping technique based on the use of a modified optical-fiber pulse compressor. The simple modifications are the substitution of a nonpolarization-preserving fiber and the addition of one polarizer. The wings of the compressed pulses were strongly reduced, and any low power satellite pulses from the laser source were also eliminated.

We acknowledge with gratitude the tireless and expert help of D. Krokel for much of the data taking. This work was partially sponsored by the U.S. Office of Naval Research.

'H. Nakatsuka, D. Grischkowsky, and A. C. Balant, Phys. Rev. Lett. 47, 910 (1981); D. Grischkowsky and A. C. Balant, Appl. Phys. Lett. 41, 1 (1982); B. Nikolaus and D. Grischkowsky, Appl. Phys. Lett. 42, 1 (1983).

${ }^{2}$ S. L. Palfry and D. Grischkowsky, Opt. Lett. 10, 562 (1985).

${ }^{3}$ A. M. Johnson, R. H. Stolen, and W. M. Simpson, Appl. Phys. Lett. 44, 729 (1984).

${ }^{4}$ J. D. Kafka, B. H. Kolner, T. Baer, and D. M. Bloom, Opt. Lett. 9, 505 (1984).

'J. P. Heritage, R. N. Thurston, W. J. Tomlinson, A. M. Weiner, and R. H. Stolen, Appl. Phys. Lett. 47, 87 (1985).

${ }^{\circ}$ C. V. Shank, R. L. Fork, R. Yen, and R. H. Stolen, Appl. Phys. Lett. 40, 761 (1982).

'J. G. Fujimoto, A. M. Weiner, and E. P. Ippen, Appl. Phys. Lett. 44,832 (1984).

${ }^{8}$ J.-M. Halbout and D. Grischkowsky, Appl. Phys. Lett. 45, 128 I (1984).

${ }^{9}$ W. H. Knox, R. L. Fork, M. C. Downer, R. H. Stolen, C. V. Shank, and J. A. Valdmanis, Appl. Phys. Lett. 46, 1120 (1985).

${ }^{10}$ R. H. Stolen, J. Botineau, and A. Ashkin, Opt. Lett. 7, 512 (1982).

"B. Nikolaus, D. Grischkowsky, and A. C. Balant, Opt. Lett. 8, 189 (1983).

${ }^{12}$ L. F. Mollenauer, R. H. Stolen, J. P. Gordon, and W. J. Tomlinson, Opt. Lett. 8, 289 (1983).

${ }^{13}$ H. G. Winful, Appl. Phys. Lett. 47, 213 (1985).

${ }^{14}$ Instead of using the standard fiber collet to hold the input end of the fiber, a 3-in. brass rod of equivalent diameter with a $90^{\circ}$ radial trough was used. The cleaved and mechanically stripped end of the optical fiber was placed in this trough and held in place with Eastman 910 adhesive. This adhesive possesses excellent mode-stripping qualities, and thereby eliminated much of the low-level light that would have otherwise propagated through this short fiber by means of the cladding modes.

${ }^{15} H$. Nakatsuka and D. Grischkowsky, Opt. Lett. 6, 13 (1981).

${ }^{16}$ We always found two orthogonal effective axes for which low power linearly polarized input light resulted in linearly polarized output light. This is not to say that the light remained linearly polarized during its total passage through the fiber. For an input polarization along an effective axis, the Soleil-Babinet compensator was not needed.

${ }^{17} R$. Ulrich and A. Simon, Appl. Opt. 18, 2241 (1979).

${ }^{18}$ W. J. Tomlinson, R. H. Stolen, and C. V. Shank, J. Opt. Soc. Am. B 1, 139 (1984).

${ }^{19}$ With a polarization preserving (strong linear birefringence) single-mode fiber, we could not observe any nonlinear birefringence under similar experimental conditions. ${ }^{13}$ 